\title{
The BEIR VII Estimates of Low-Dose Radiation Health Risks Are Based on Faulty Assumptions and Data Analyses: A Call for Reassessment
}

\author{
Jeffry A. Siegel ${ }^{1}$, Bennett S. Greenspan ${ }^{2}$, Alan H. Maurer ${ }^{3}$, Andrew T. Taylor ${ }^{4}$, William T. Phillips ${ }^{5}$, Douglas Van \\ Nostrand $^{6}$, Bill Sacks ${ }^{7}$, and Edward B. Silberstein ${ }^{8}$ \\ ${ }^{I}$ Nuclear Physics Enterprises, Marlton, New Jersey; ${ }^{2}$ Department of Radiology, Medical College of Georgia at Augusta University, \\ Augusta, Georgia; ${ }^{3}$ Temple University School of Medicine, Philadelphia, Pennsylvania; ${ }^{4}$ Emory University School of Medicine, Atlanta, \\ Georgia; ${ }^{5}$ Department of Radiology, University of Texas Health Science Center at San Antonio, San Antonio, Texas; ${ }^{6}$ Nuclear Medicine \\ Research, MedStar Health Research Institute, Washington, District of Columbia; ${ }^{7}$ U.S. Food and Drug Administration (retired), Green \\ Valley, Arizona; and ${ }^{8}$ Departments of Radiology and Medicine (emeritus), University of Cincinnati Medical Center, Cincinnati, Ohio
}

See an invited perspective on this article on page 1014.

The 2006 National Academy of Sciences Biologic Effects of lonizing Radiation (BEIR) VII report is a well-recognized and frequently cited source on the legitimacy of the linear no-threshold (LNT) model-a model entailing a linear and causal relationship between ionizing radiation and human cancer risk. Linearity means that all radiation causes cancer and explicitly excludes a threshold below which radiogenic cancer risk disappears. However, the BEIR VII committee has erred in the interpretation of its selected literature; specifically, the in vitro data quoted fail to support LNT. Moreover, in vitro data cannot be considered as definitive proof of cancer development in intact organisms. This review is presented to stimulate a critical reevaluation by a BEIR VIII committee to reassess the validity, and use, of LNT and its derived policies.

Key Words: BEIR VII; linear no-threshold; radiological imaging

J Nucl Med 2018; 59:1017-1019

DOI: 10.2967/jnumed.117.206219

$\mathbf{T}$ he National Academy of Sciences Biologic Effects of Ionizing Radiation (BEIR) VII report (1) concludes that the available human epidemiologic studies and animal experiments lack statistical power to distinguish a linear no-threshold (LNT) model from the absence of an effect at lower doses. Although none of the numerous publications cited in BEIR VII have demonstrated increased cancer risk from low-dose radiation exposure and thus none provide evidence for LNT, BEIR VII nevertheless asserts that the current scientific evidence is consistent with a linearly proportional relationship between cancer risk and low-dose ionizing radiation and that there is no threshold below which an increased cancer risk does not occur.

On page 245 of BEIR VII, the report asserts,

It is abundantly clear that direct epidemiologic and animal approaches to low-dose cancer risk are intrinsically limited in their capacity to define possible curvilinearity or dose thresholds for risk in the range

Received Dec. 18, 2017; revision accepted Feb. 12, 2018.

For correspondence or reprints contact: Jeffry A. Siegel, Nuclear Physics Enterprises, 4 Wedgewood Dr., Marlton, NJ 08053.

E-mail: nukephysics@comcast.net

Published online Feb. 23, 2018.

COPYRIGHT (C) 2018 by the Society of Nuclear Medicine and Molecular Imaging.
0-100 mSv. For this reason the present report has placed much emphasis on the mechanistic data that can underpin such judgments.

BEIR VII's mechanistic view subscribes to a model of radiation carcinogenesis consisting of a series of events in intact organisms - from radiation exposure to different types of DNA damage followed by chromosomal damage and (incomplete) repair to clinical cancer-leading inevitably to clinical cancer. Since BEIR VII notes that epidemiologic data linking low-dose radiation to clinical cancer are "intrinsically limited," much emphasis is placed on a single study concerning one "mechanistic" feature of cancer initiation, that of radiogenic chromosomal damage. That study (2), performed in vitro, was selected to better define the shape of the dose-response curve in the low-dose range of $0-100 \mathrm{mSv}$ and is the focus of the current review.

Also on page 245 the report says,

...given the intimate relationship established between DNA damage response, gene or chromosomal mutations, and cancer development, the form of the dose-response for mutation induction in single cells should be broadly informative for cancer initiation. Data from a largescale study noted in Chapter 2 suggest a linear relationship between low-[linear energy transfer] dose and chromosomal mutation down to around $20 \mathrm{mGy}$.

The large-scale study is by Lloyd et al. (2) and is the basis of BEIR VII's assertion:

Considerable effort has been expended to test this proposition [i.e., that the dose-response for all forms of mutations will be linear at low doses and low dose rates], and in a very large multicenter study using assays of dicentric aberrations [one biomarker of radiation damage] in human lymphocytes, the linearity of the response was evident down to at least $20 \mathrm{mGy}$ of low-[linear energy transfer] radiation (Lloyd and others 1992), which is illustrated in Figure 2-5 [reproduced as Fig. 1]. Below that dose, the statistical power of the data was not sufficient to exclude the theoretical possibility of a dose threshold for radiation effects.

Lloyd's study is a collaboration among 6 laboratories that counted chromosomal aberrations in human lymphocytes induced in vitro by $\mathrm{x}$-rays over a 0 - to $300-\mathrm{mGy}$ dose range. Figure 1 is an exact replication of BEIR VII's Figure 2-5, which reproduces a figure from the Lloyd article (2). Figure 1 represents 3 datasets from separate experiments, two by Lloyd and one by Pohl-Rüling et al. (3). For better clarity, we have redrawn Figure 1 as a bar graph, with a nonlinear $x$-axis, in Figure 2, showing only mean values of dicentrics at each nominal dose level and Lloyd's 


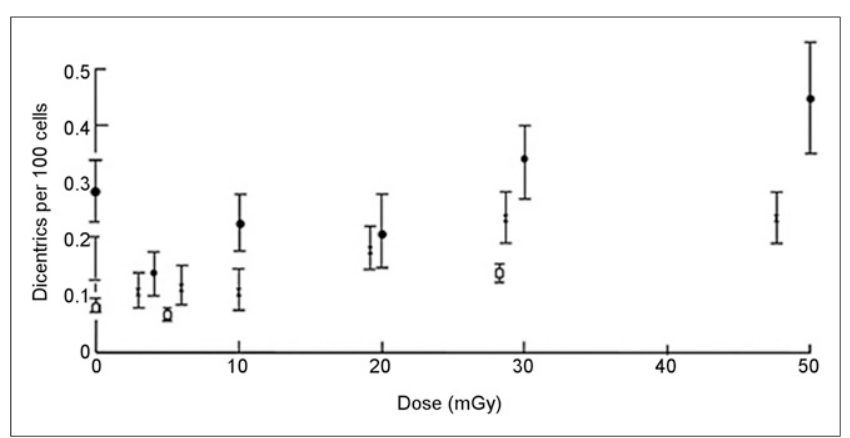

FIGURE 1. BEIR VII's Figure 2-5, showing dicentric yields as function of dose: Pohl-Rüling et al. (3) (•); experiment 1 of Lloyd et al. (2) (x); experiment 2 of Lloyd et al. (2) ( $\square$ ).

zero-dose data point of 0.17 for experiment 1 , which was omitted from BEIR's Figure 2-5.

\section{CRITIQUE OF USE OF IN VITRO MECHANISTIC DATA}

Specifically, in experiment 1 , Lloyd et al. found the numbers of dicentrics per 100 cells to be $0.17,0.11,0.12,0.11,0.19,0.24$, 0.24 , and 1.28, for nominal doses of $0,3,6,10,20,30,50$, and 300 mGy, respectively (Figs. 1 and 2). Relative to the control (zerodose) value, Lloyd found actual reductions in dicentrics at the nominal doses of 3, 6, and $10 \mathrm{mGy}$, followed by small increases at the higher doses of $20,30,50$, and a significant increase only at the much higher dose of $300 \mathrm{mGy}$. In Lloyd's experiment 2, fewer doses were used, but the data support this initial reduction in dicentrics from the control value. Nevertheless, they dismiss the values below $20 \mathrm{mGy}$ as subject to too much uncertainty based on the small numbers of damaged dicentrics, saying that the apparent reduction at the 3 lowest nonzero doses most likely, "is not of biological origin but may be an artefact of the higher-than-expected frequency of spontaneously arising [zero-dose] aberrations."

Lloyd et al. rejected the "higher-than-expected" zero-dose value as valid, interpreting it as statistical noise, and because of statistical variations below $20 \mathrm{mGy}$, concluded that, "the question of whether linearity extends down to zero dose, or whether there is a plateau (threshold) indicative of inducible repair remains open." This served as justification for their assertion that, "there is nothing in the present work which is incompatible with the prudent radiological protection assumption of a linear initial slope."

In fact, the data are not compatible with a "linear initial slope." There is no initial linear rise; rather, there is no measurable

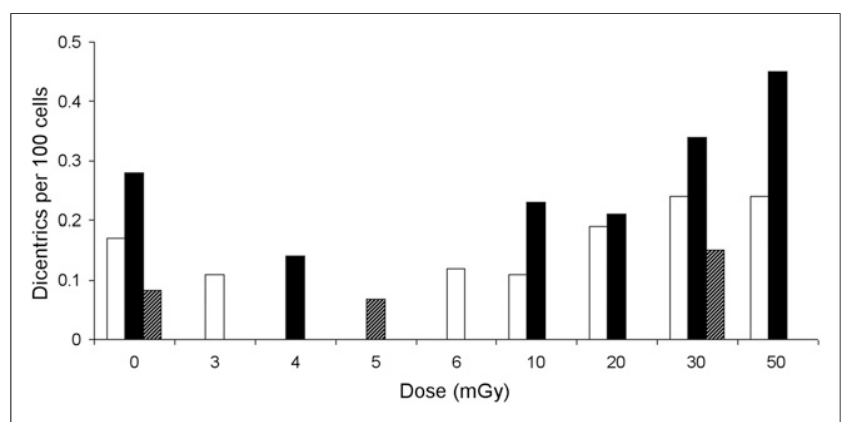

FIGURE 2. Bar graph representation of BEIR VII's Figure 2-5. White bars are data from experiment 1 of Lloyd et al. (2); striped bars are data from experiment 2 of Lloyd et al. (2); black bars are data from study of Pohl-Rüling et al. (3). increased dose response from 3 to $10 \mathrm{mGy}$ and all these initial values are no greater than the zero-dose value. Above $10 \mathrm{mGy}$, there is a less-than-proportional (i.e., nonlinear) rise and possibly a second plateau from 20 to $50 \mathrm{mGy}$ (Figs. 1 and 2), in the range where Lloyd regards the data as less uncertain and more reliable. These data, in contrast, appear more supportive of a threshold. Lloyd also cites a previous, similar in vitro study by Pohl-Rüling et al. (3), who found a similar initial negative slope but, rather than dismissing the finding as noise, offered a biologic explanation (see below). Pohl-Rüling's data are also represented in BEIR VII's Figure 2-5, albeit without mention in the report.

With respect to the 20- to $300-\mathrm{mGy}$ data points in experiment 1 , Lloyd concludes that, "This work has shown conclusively that linearity of biological effect can be demonstrated with this system down to doses of about 20 mGy." However, this is not borne out by their data. The inclusion of the non-low-dose ( $300 \mathrm{mGy}$ ) value dominates the increase in dicentrics above $20 \mathrm{mGy}$, and the departure from linearity at 30 and $50 \mathrm{mGy}$ was implicitly regarded as measurement error, although Lloyd downplays error in this range. Not only are the 20- to 50-mGy values relatively flat, but they hardly exceed the empiric control value $(0.17)$ at $0 \mathrm{mGy}$.

Furthermore, Lloyd's data are consistent with a more recent observation from the Life Span Study atomic bomb survivor population (4):

Linearity at low doses does not exist [in this case 20-50 mGy and likely beyond, somewhere between 50 and $300 \mathrm{mGy}$; rather, it is forced by the high-dose extrapolation of the LNT model.

In contrast to Lloyd, Pohl-Rüling does not discount the initial reduction in the dicentrics below the control value (at doses of 4 , 10, and even up to $20 \mathrm{mGy}$; Figs. 1 and 2). In agreement with many other experimenters who have found the same feature $(5,6)$, Pohl-Rüling acknowledges the reductions as being real and offers the following biologic explanation:

The deviations can be explained by an altered kinetics of aberration production at very low doses probably due to DNA repair mechanisms operating in these cells.

\section{DISCUSSION}

BEIR VII, first, claims “a linear relationship between low-linear energy transfer dose and chromosomal mutation down to around 20 mGy" - an assessment not established by Lloyd's data-and, second, ignores the fact that Lloyd's data were obtained only in vitro. Such studies can only suggest mechanisms of cancer initiation; they cannot by themselves provide evidence of clinical cancer development in whole organisms. In fact, on page 133 of Chapter 5, the BEIR report asserts that "at relatively low doses, there is still uncertainty as to whether there is an association between radiation and disease, and if there is an association, there is uncertainty about whether it is causal or not."

Thus, BEIR's assertion that the link between initiation and clinical cancer has been "established" is unsupportable. The continual creation from normal mitochondrial metabolism, in both intact organisms and cells in culture, of DNA-damaging reactive oxygen species results in frequent production of genetic mutations, but clinical cancer development in intact organisms is usually prevented by evolved adaptive protective responses, from cellular (described by Pohl-Rüling) to tissue to organismal levels. These mechanisms include antioxidant production, bystander signaling among damaged cells and their neighbors, and immune system cleanup of unrepaired cells (5-7). 
Although DNA damage (i.e., genetic mutations) may be a necessary prequel, it is far from sufficient to produce clinical cancer $(8,9)$. The outdated "one mutation $=$ one cancer" model has been replaced by immune-system failure (4). Subsequent repair or removal of DNA double-strand breaks back to background levels have been demonstrated in patients within $24 \mathrm{~h}$ after a CT scan (10).

BEIR VII's assertion - that "the presence of a true dose threshold demands totally error-free" repair in response to radiogenic damage-ignores removal responses. And their own selected data from Lloyd (and incidentally the data of Pohl-Rüling) negate the essential conclusion of their report that "current scientific evidence is consistent with the hypothesis that there is a linear, no-threshold dose-response relationship between exposure to ionizing radiation and the development of radiation-induced solid cancers in humans." Specifically, the Lloyd study negates LNT on both counts; it fails to show either linearity from zero-dose or absence of a threshold. This serves to confirm the significant evidence that our bodies do not respond similarly to high- and low-dose exposure (4-7).

BEIR VII is probably the most frequently cited support for LNT, but there are serious concerns that undermine its validity as a guide to either radiation science or regulatory policies. The resulting LNT-based risks currently associated with low radiation doses appear to be overestimated and fail to reflect the actual riskbenefit balance of medical imaging.

\section{CONCLUSION}

Nuclear medicine physicians, radiologists, and radiation protection professionals aim to convey accurate radiation-related information to patients and the public, but their reliance on BEIR VII is unfortunately based on flawed interpretation of the BEIR VII Committee's selected literature. The consequences of misdiagnoses due to imaging avoidance are potentially more immediate and harmful than any future LNT-predicted cancers avoided by stringent dose-reduction strategies. For these reasons, we call for the establishment of a BEIR VIII committee to critically reassess the validity, and use, of LNT and its derived policies.

\section{DISCLOSURE}

The opinions expressed in this article are the personal opinions of the authors. No other potential conflict of interest relevant to this article was reported.

\section{REFERENCES}

1. National Research Council of the National Academies. Health Risks from Exposure to Low Levels of Ionizing Radiation: BEIR VII Phase 2. Washington, DC: The National Academies Press; 2006:245.

2. Lloyd DC, Edwards AA, Leonard A, et al. Chromosomal aberrations in human lymphocytes induced in vitro by very low doses of x-rays. Int J Radiat Biol. 1992;61:335-343.

3. Pohl-Rüling J, Fischer O, Haas G, et al. Effect of low-dose acute $\mathrm{x}$-irradiation on the frequencies of chromosomal aberrations in human peripheral lymphocytes in vitro. Mutat Res. 1983;110:71-82.

4. Siegel JA, Welsh JS. Does imaging technology cause cancer? Debunking the linear no-threshold model of radiation carcinogenesis. Technol Cancer Res Treat. 2016;15:249-256.

5. Luckey TD. Radiation Hormesis. Boca Raton, FL: CRC Press; 1991.

6. Aurengo A, Averbeck D, Bonnin A, et al. Dose Effect Relationships and Estimation of the Carcinogenic Effects of Low Doses of Ionizing Radiation. Paris, France: Académie des Sciences-Académie nationale de Médecine; March 2005.

7. Sacks B, Meyerson G, Siegel JA. Epidemiology without biology: false paradigms, unfounded assumptions, and specious statistics in radiation science (with commentaries by Inge Schmitz-Feuerhake and Christopher Busby and a reply by the authors). Biol Theory. 2016;11:69-101.

8. Siegel JA, Pennington CW, Sacks B. Subjecting radiological imaging to the linear no-threshold hypothesis: a non sequitur of non-trivial proportion. $\mathrm{J} \mathrm{Nucl}$ Med. 2017;58:1-6.

9. Siegel JA, Sacks B, Pennington CW, Welsh JS. Dose optimization to minimize radiation risk for children undergoing $\mathrm{CT}$ and nuclear medicine imaging is misguided and detrimental. J Nucl Med. 2017;58:865-868.

10. Löbrich M, Rief N, Kühne M, et al. In vivo formation and repair of DNA double strand breaks after computed tomography examinations. Proc Natl Acad Sci USA. 2005;102:8984-8989. 